\title{
A Stochastic knapsack problem with mixed distributional capacity
}

\author{
E. H. Etuk, I. D. Essi and N.P. Akpan \\ Dept. of Mathematics/Computer Science, Rivers State University of Science and Technology, \\ Port Harcourt, Nigeria \\ etteuk@yahoo.com, didiessi@yahoo.com,nsigreat@yahoo.com
}

\begin{abstract}
We present a stochastic knapsack problem with the capacity following a mixed distribution. The problem is an optimization type where one has to maximize the profit of the content of the knapsack without exceeding the capacity of the knapsack and as such the cost is minimized. A mixture of Exponential and Poisson distribution was applied using the multiplicative approach of Mood \& Graybill (2006).
\end{abstract}

Keywords: knapsack, stochastic, mixed distribution

\section{INTRODUCTION:}

This work focuses on an aspect of knapsack problem where the parameter of interest, the capacity is of the mixed distribution. It is our observation that researchers have done so much on the other parameters (the profit and the weight) to be either of continuous random type or of discrete type. Recently, Witchakul \& Ayudthaya (2008) observed that the capacity of the knapsack has been neglected, whereas studying uncertainty in capacity is also important. Their idea was based on the fact that in cargo loading problem, when the truck has many customers to load the items. The available space is unknown before the truck arrives. Hence the remaining capacity of the truck is uncertain. Whereas our motivation was on the series of activities going on in a processing Mill (a multipurpose Mill). Here, both cassava is grinded for processing garri, palm fruits are processed for the production of palm oil, grains are being grinded etc. Note that all these activities are processed by one engine with different attachments but it is operated by one operator. It is observed that customers arrives randomly and the operator has a limited time for accepting customers and also the capacity of the shop is equally considered such that customers items will not be kept outside for security reasons. A penalty will accrue for customer's freewill.

Mixed distribution: This work is based on the assumption that a random variable $X$ takes up distinct values $x_{1}, x_{2}, \ldots, x_{n}$ with positive probabilities and also takes up (assume) all values interval; say $a \leq X \leq b$. The probability distribution that will be obtained here will be as the result of the combination of all discretes, or all continuous or both discrete and continuous distribution. This process constitutes what is termed mixed distribution or contagious distribution. For instance, this combination for mixed distribution can be either additive or multiplicative. It can be of discrete distribution, continuous distribution or even a combination of the two distributions as earlier mentioned. Literature has shown that much work has not been done in this area. Mood et al(1963) stated; if $f_{0}(),. f_{1}(),. \ldots, f_{n}(),. \ldots$ is a sequence of density functions which are either all discrete density functions or all probability density functions which may or may not depend on parameters and $P_{0}, P_{1}, \ldots, P_{n}$, is a sequence of parameters satisfying $P_{i} \geq 0$ and $\sum_{i=0}^{\infty} P i=1$, then $\sum_{i=0}^{\infty} P i f i(x)$ is a density function, which is sometimes called a contagious or mixture. For example if $\mathrm{f}_{0}(\mathrm{x})=\Phi \mu_{0} \delta_{0}^{2}(x)$ (a normal with mean $\mu_{0}$ and variance $\left.\delta_{0}^{2}\right)$ and $\mathrm{f}_{1}(\mathrm{x})=$ $\Phi \mu_{1} \delta_{1}^{2}(x)$ then

$P_{0} \Phi \mu_{0} \delta_{0}^{2}(x)+P_{1} \Phi \mu_{1,} \delta_{1}^{2}(x)=$

$(1-P) \frac{1}{\sqrt{2 \pi \delta_{0}}} \ell^{-\frac{1}{2}\left[\left(X-\mu_{0}\right) / \delta_{0}\right]^{2}}+$

$P \frac{1}{\sqrt{2 \pi \delta_{1}}} \ell^{-\frac{1}{2}\left[\left(X-\mu_{1}\right) / \delta_{1}\right]^{2}}$ 
Where $P_{1}=P$ and $P_{0}=1-P$, is a mixture of two normal densities.

He further stated the extension of a mixed distribution as; Let $\{f(x ; \theta)\}$ be a family of density functions parameterized or indexed by $\theta$. Let the totality of values that the parameter $\theta$ can assume be denoted by $\Theta$. If $\Theta$ is an interval (possibly infinite) and $g(\theta)$ is a probability density function which is zero for all arguments not in $\Theta$, then

$\int_{\Theta} f(x ; \theta) g \theta d \theta$

Is a density function, called a mixed distribution. An example was a mixture of poison and gamma distribution.

$$
\begin{aligned}
f(x ; \theta)= & \ell^{-\theta} \theta^{x} / x ! \quad \text { For } x=0,1,2, \ldots \\
& 0 \text { otherwise }
\end{aligned}
$$

$$
\begin{aligned}
& \text { and } g(\theta)=\frac{\lambda^{r}}{\sqrt{(r)}} \theta^{r-1} \ell^{-\lambda \theta} \text { then } \int_{0}^{\infty} f(x ; \theta) g \theta d \theta \\
& =\int_{0}^{\infty} \frac{\ell^{-\theta} \theta^{x}}{x !} \cdot \frac{\lambda^{r}}{\Gamma(r)} \theta^{r-1} \ell^{-\theta \lambda} d \theta
\end{aligned}
$$

The above illustration supports the additive of two continuous distribution and the multiplicative of a discrete and continuous random variables.

Also, Stirzaker (1994) defines his mixed distribution as let $f_{1}(x)$ and $f_{2}(x)$ be density functions, and let

$$
f_{3}(x)=\lambda f_{1}(x)+(1-\lambda) f_{2}(x)
$$

Where

$$
\begin{aligned}
& 0 \leq \lambda \leq 1 \\
& \text { Then } \\
& f_{3}(x) \geq 0 \\
& \text { and } \\
& \int f_{3}=\lambda \int f_{1}+1-\lambda \int f_{2}=1
\end{aligned}
$$

Hence $f_{3}$ is a density and is said to be a mixture of $f_{1}$ and $\mathrm{f}_{2}$.

There are other related literature concerning mixed distribution: Mood et al (2006), Meyer (1965), Feller(1970) etc.

Related literature: Quandt and Ramsey (1978) as cited by Greene (2003) analyzed the problem of estimating the parameters of a mixture of normal distributions.

Raynal and Gelevara, (1997) mixed two Gumbel distributions in other to model annual floods (NERC, 1975) then the five parameters were obtained.

In the case of knapsack problem, much work has been done in this area since the area is broad and has many practical applications. Witchakul and Ayudthaya (2005) pointed out that the principal approaches for solving a knapsack problem are; Dynamic programming, Network approaches, Enumeration Algorithms and Branch and Bound approaches. Bellman (1957) observed that Dynamic programming is not an efficient way to solve a large size knapsack problem. Cabot (1970) applied enumeration algorithm to a knapsack problem. Mathur (1980) proposed an enumeration algorithm for the general multiple choice knapsack problem while Shapiro (1968) formulated a knapsack problem as a shortest route problem. However, this method is not popular because it requires enormous number of nodes and arcs.

There are three main versions of knapsack problem; the unbounded knapsack problem, the bounded knapsack problem and the $0-1$ knapsack problem. Dantzig (1957) was the first person that presented the first upper bound for knapsack, based on a continuous relaxation; see Witchakul and Ayudthaya (2005). Meanwhile, there are many researchers that have mentioned the branch and bound algorithm for solving 0 - 1 knapsack problem; Kolesar (1967), Horowitz and Sahni (1974), Fayard and Plateau (1975), Nauss (1976), Martello and Toth $(1977,1988)$ and the rest.

Balas and Zemel (1980) in their work on an Algorithm for large $0-1$ knapsack problems pointed out that this algorithm relies mainly on three main ideas. The first idea is to focus on core problem. The second one is binary search method without sorting variables and the last one is a simple heuristic whose accuracy 
improves exponentially with problem size. Ram and Sarin (1988) described branch and bound algorithm for the $0-1$ equality knapsack problem. Volgenant (1998) applied core approach to the $0-1$ equality knapsack problem and compared with Ram and Sarin algorithm. They have shown that core approach is better in solution time.

Capital budgeting problem is also referred to as the multidimensional knapsack problem in the literature, Murty (1995). Benli and Bilici (2006) stated that the capital budgeting problem is one of the first integer programming problems studied, and that it was first posed by Lorie and Savage (1955) as:

$$
\begin{aligned}
& \operatorname{Max}=\sum_{j=1}^{n} c_{j} x_{j} \\
& \text { Subject to } \sum_{j=1}^{n} a_{j} x_{j} \leq \mathrm{b} \\
& \mathrm{x}_{\mathrm{j}} \in\{0,1\} \mathrm{j}=1,2, \ldots, \mathrm{n}
\end{aligned}
$$

Where $n$ projects are under consideration and $C_{J}$ is the net present value of project $j$, and $a_{j}$ is the capital required to fund project $j$. The total capital available for all projects is $b$. The decision variable, $x_{j}$, is equal to 0 if project $j$ is not selected and 1 if it is selected to be funded.

Weingartner (1963) analyzed the above 'Lorie Savage problem' and established a frame work for the capital budgeting problems. Following his pioneering work, an extensive literature was developed on the mathematical approaches to capital budgeting problems using linear programming; Bernhard (1969), Weingartner (1966), Freeland and Ronsenblatt (1978), Myers (1972), Park and SharpBette (1990). And for other reviews on approaches to capital budgeting problem, including goal programming, nonlinear programming, mixed integer programming and simulation, see Benli and Bilici (2006).

Munapo (2007) proposed a special algorithm to solve the capital budgeting model. Kress et al (2007) presents a new combinatorial problem, called Mimmax multidimensional knapsack problem (MKP), motivated by a military logistic problem. They further show that the MKP is a NP - hard and developed a practically efficient and combinatorial algorithm for solving it. Bean et al (1987) solved a multi-period version of the problem where the objective is to maximize net cash present value profit by divesting assets subject to certain lower bounds on the return on equity that companies must achieve each year. Also, Hall et al (1992) formulated an integer programming problem. The problem was to decide on project funding at a National cancer institute of USA. Other recent developments in the area of integer programming as cited by Munapo(2007) are Gomory et al (2003), who applied corner polyhedral as a technique to solve the integer model. Kumar et al (2007) used the non basic variables to descend to the optimal integer solution from the continuous optimal point and they also proposed a technique for significantly reducing the complexity of the general integer model; Kumar et al (2007).

Literature has also shown that the study of stochastic knapsack problem is limited almost exclusively to two cases in which the value of the objects themselves arrive as part of a stochastic process. A detailed coverage of these problems can be found in Caraway et al (1993), Steinberg and Parks (1979), Henig (1990), Morita et al (1989), Papastravrou et al (1996), Tamaki (1986), Righter (1989), Ross and Tsang (1989); see Kleywegt and Papastavrou (1998). Balas and Zemel (1980), proposed an algorithm for large knapsack problems. They have been the first to propose an algorithm in linear time to solve the linear programming relaxation of the knapsack problem. The multidimensional knapsack problem is mostly applicable in resource allocation problem; Wilbaut et al (2008).Wilbaut et al(2008) further stated that, Similar work can be found in Lorie and Savage (1955), Weingartner(1966), Weingartner and Ness(1967) and Manne and Markowitz (1957). Vasquez and Hao (2001a) used the multidimensional knapsack problem to model the daily management of satellite like spot.

\section{Assumptions:}

1) The desired variable $x_{i}$ is limited

2) Weight $\left(\mathrm{w}_{\mathrm{i}}\right)>0$ and $\operatorname{cost}\left(\mathrm{C}_{\mathrm{i}}\right)>0$

3) $0<\frac{C_{1}}{w_{1}} \leq \frac{C_{2}}{w_{2}} \leq \ldots \leq \frac{C_{n}}{w_{n}}$

4) There is a time limit for accepting items from the customers

5) Customers arrival is stochastic and the weight (quantity) of items is not known until it arrival.

6) Items may be accepted or rejected 
A mixture of Poisson and Exponential Distribution: Let us assume that the capacity of the knapsack follows a mixture of Poisson and Exponential distribution, thus :

Using $\int_{\theta} f(x ; \theta) g(\theta) d \theta$

Let $f(x ; \theta)=\frac{\ell^{-\theta} \theta^{x}}{x !} \quad \mathrm{x}=0,1,2, \ldots$

Let $g(\theta)=\theta \ell^{-\theta x} \quad ; \mathrm{x}>0$

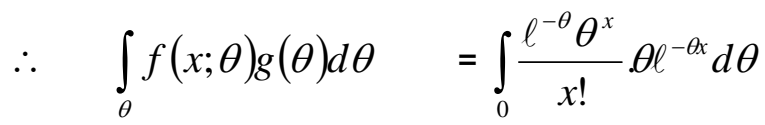

$=\int_{0}^{\infty} \frac{\theta^{x+1}}{x !} \ell^{-(1+x) \theta} d \theta=\frac{1}{x !} \int_{0}^{\infty} \theta^{x+1} \ell^{-(1+x) \theta} d \theta$

$=\frac{1}{x !} \int_{0}^{\infty} \theta^{x+2-1} \ell^{-(1+x) \theta} d \theta=$

$\frac{\Gamma(x+2)}{x !(x+1)^{x+2}} \int_{0}^{\infty} \frac{(x+1)^{x+2} * \theta^{(x+2)-1} \ell^{-(1+x) \theta}}{\Gamma(x+2)} d \theta$

$=\frac{\Gamma(x+2)}{x !(x+1)^{x+2}} \int_{0}^{\infty} \frac{(x+1)^{x+2} \theta^{(x+2)-1} \ell^{-(1+x) \theta}}{\Gamma(x+2)} d \theta$

Since $\int_{0}^{\infty} \frac{(x+1)^{x+2}}{\Gamma(x+2)} * \theta^{(x+2)-1} \ell^{-(x+1) \theta} d \theta=1$
Therefore $\int_{0}^{\infty} \frac{\ell^{-\theta} \theta^{x}}{x !} * \theta \ell^{-\theta x} d \theta=\frac{\Gamma(x+2)}{x !(x+1)^{x+2}}$

$=\frac{\Gamma(x+2)}{x !(x+1)^{x}(x+1)^{2}}$ which may be written as

$\frac{\Gamma(x+2)}{x !}\left(\frac{1}{x+1}\right)^{x}\left(\frac{1}{x+1}\right)^{2} ; \mathrm{x}=0,1,2, \ldots$

The graph of the distribution: The values for the distribution where generated by substituting the respective values of $x=0,1,2,3,4$ and 5 . But recall that the gamma function, denoted by $\Gamma($.$) is defined by$

$\Gamma(t)=\int_{0}^{\infty} x^{t-1} \ell^{-x} d x$ for $t>0$

$\Gamma(t)$ is nothing more than a notation for the definite integral that appears on the right hand side of the above equation. Integration by parts yields $\Gamma(t+1)=$ $t \Gamma(t)$ and, hence, if $\mathrm{t}=\mathrm{n}$ (an integer), $\Gamma(t+1)=\mathrm{n}$ ( see Mood and Graybill (2006))

\section{A graph showing the mixture of exponential and Poisson distribution}

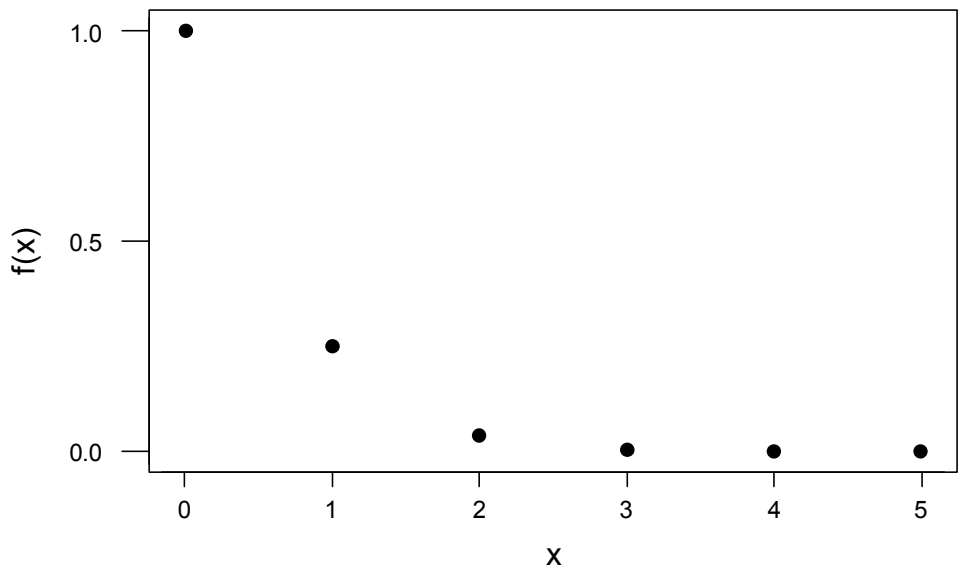




\section{METHODS:}

In this paper we propose a strategy and algorithm for solving stochastic knapsack problem with a mixed distribution probability on the capacity. The problem is a minimization case, as such we add the non negative variables (slack variable) and subtract the non negative variable (surplus variable) so that we can work with the equality sign instead of the inequality sign. Going by the nature of the problem we are trying to solve, there are three possible situations that can affect the capacity of the knapsack

1) A case where the sum of weights of the items are greater than or equal to the capacity

$$
\begin{array}{ll}
\text { Min } \mathrm{f} & =\sum_{i=1}^{n} C_{i} x_{i} \\
\text { S.t } & \sum_{i=1}^{n} w_{i} x_{i} \geq k
\end{array}
$$

2) A case where the sum of weights of the items are less than or equal to the capacity

$$
\begin{gathered}
\text { Min } \mathrm{f}=\sum_{i=1}^{n} C_{i} x_{i} \\
\text { S.t } \quad \sum_{i=1}^{n} w_{i} x_{i} \leq k
\end{gathered}
$$

3) A case where the sum of weight of the items are strictly equal to the capacity

$$
\begin{aligned}
& \text { Min } \mathrm{f}=\sum_{i=1}^{n} C_{i} x_{i} \\
& \text { S.t } \sum_{i=1}^{n} w_{i} x_{i}=k \\
& C_{i} \text { is the cost coefficient of the desired } \\
& \mathrm{X}_{\mathrm{i}} \quad \text { is the desired variable } \\
& \mathrm{W}_{\mathrm{i}} \text { is the weight coefficient of the desired }
\end{aligned}
$$

Where $\quad C_{i}$ is the cost coefficient of the desired variable

variable

$\mathrm{K}$ is the capacity of the knapsack
However, we observe that the first case has an inequality sign $(\geq)$ in its constraints. This can be converted to an equality sign by introducing the nonnegative variable (the surplus) variable to it and our new constraints will be

$$
\sum_{i=1}^{n} w_{i} x_{i}-s_{i}=k
$$

Also, going by the same arguments, the constraint for the second case will be

$$
\sum_{i=1}^{n} w_{i} x_{i}+s_{i}=k
$$

Where $S_{i}$ is the slack variable.

A case where we are using a mixed constraint

$$
\text { Optimize } \mathrm{Z}=\sum_{i=1}^{n} C_{i} x_{i}
$$

$$
\begin{aligned}
& \text { S.t } \quad \sum_{i=1}^{n} w_{i} x_{i} \geq k \\
& \sum_{i=1}^{n} w_{i} x_{i} \leq k \\
& \sum_{i=1}^{n} w_{i} x_{i}=k
\end{aligned}
$$

$x_{i} \geq 0 \quad \mathrm{i}=1,2, \ldots, \mathrm{n}$

Will be transformed to

Optimize $\mathbf{Z}=\sum_{i=1}^{n} C_{i} x_{i}+\sum_{i=1}^{n} 0 . S_{i}+\sum_{i=1}^{n} 0 . S_{i}$

$$
\begin{array}{r}
\text { S.t } \quad \sum_{i=1}^{n} w_{i} x_{i}-s_{i}=k \\
\sum_{i=1}^{n} w_{i} x_{i}+s_{i}=k
\end{array}
$$


$\sum_{i=1}^{n} w_{i} x_{i}=k \quad x_{i} \geq 0 \quad ; \quad \mathrm{i}=1,2, \ldots, \mathrm{n}$

Where the coefficients of slack and surplus variables are zero in the objective function due to the reason that they represent unused capacity or resource. See (Sharma2004)

Our proposed model will deviate a little from this, since we are considering a case of mixed distribution. Hence our objective function is stated as

Minimize $f=$

$\sum_{i=1}^{n} C_{i} x_{i}+g \int_{L}^{U} P(k) u(k) d k+h \int_{L}^{U} P(k) v(k) d k$

S.t $\quad \sum_{i=1}^{n} w_{i} x_{i}+u(k)-v(k)=k$

$$
L \leq K \leq U \quad ; \mathrm{U}(\mathrm{k}) \cdot \mathrm{V}(\mathrm{k})=0 \quad ;
$$

$\int P(k) d k=1$

$x_{i} \geq 0$ and integer

Where $U(k)$ is the slack variable with the capacity $k$

$\mathrm{V}(\mathrm{k})$ is the surplus variable with respect to the capacity $k$ capacity $\mathrm{k}$

$P(k)$ is the probability distribution of the

$L$ is the lower bound of the capacity

$U$ is the upper bound of the capacity

$g$ is the penalty cost of the slack variable

$h$ is the penalty cost of the surplus variable

we defined the interval $Q \in[L, U]$

there exist $\sum_{i=1}^{n} w_{i} x_{i} \geq Q$ and

$$
\sum_{i=1}^{n} w_{i} x_{i}<Q+\Delta Q
$$

Where $Q$ is a positive real number. Let

$$
\begin{aligned}
& \sum_{i=1}^{n} w_{i} x_{i}=\alpha \\
& \alpha \geq Q \quad ; \alpha<Q+\Delta Q
\end{aligned}
$$

Hence $Q$ creates a neighborhood for $Q=\sum_{i=1}^{n} w_{i} X_{i}$

Define $\mathrm{V}(\mathrm{k})=\sum w_{i} x_{i}-k \quad L \leq k<Q$

$$
\mathrm{U}(\mathrm{K})=K-\sum w_{i} x_{i} \quad Q<k \leq U
$$

Substituting these into our objective function we have $\operatorname{Min} \mathrm{f}=$

$\sum_{i=1}^{n} C_{i} x_{i}+g \int_{Q}^{U} P(k)\left(k-\sum_{i=1}^{n} w_{i} x_{i}\right) d k+h \int_{L}^{Q} P(k)\left(\sum_{i=1}^{n} w_{i} x_{i}-k\right) d k$ $=$

$\left.\left.\sum_{i=1}^{n} G X_{i}+g \int_{Q}^{U} k(f) d k-g \int_{Q}^{U} P(k) \sum_{i=1}^{n} w_{i} X_{i}\right) d k+h \int_{L}^{Q} P(k) \sum_{i=1}^{n} W_{i} X_{i}\right) d k-h \int_{L}^{Q} k(f k) d l$

S.t $\quad \sum_{i=1}^{n} w_{i} x_{i} \geq Q$

$$
\sum_{i=1}^{n} w_{i} x_{i}<Q+\Delta Q
$$

Relaxation and upper bound: The most natural, and historically the first, relaxation of knapsack problem is the linear programming relaxation, that is the continuous knapsack problem obtained from

$$
\begin{aligned}
& \text { Maximize } Z=\sum_{j=1}^{n} p_{j} x_{j} \\
& \text { S.t } \quad \sum_{j=1}^{n} w_{j} x_{j} \leq c \\
& \quad x_{j}=0 \text { or } 1, \quad j \in N=\{1,2, \ldots, n\}
\end{aligned}
$$


Where $x_{j}=\left\{\begin{array}{cc}1 & \text { if } \\ 0 & \text { otherwise }\end{array}\right.$

By removing the integrality constraint on $x_{j}$ :

Maximize $\mathrm{Z}=\sum_{j=1}^{n} p_{j} x_{j}$

S.t $\quad \sum_{j=1}^{n} w_{j} x_{j} \leq c$

$0 \leq x_{j} \leq 1, \quad \mathrm{j}=1, \ldots, \mathrm{n} \quad$ (see Martello \&Tooth 1990)

We assume that the items are ordered such that the cost per unit weight increases monotonically i.e

$$
\frac{c_{1}}{w_{1}} \leq \frac{c_{2}}{w_{2}} \leq \ldots \leq \frac{c_{n}}{w_{n}}
$$

These items are consecutively inserted into the knapsack until the first item, s, is found which cannot be included into the knapsack. We call it the critical item i.e

$$
\mathrm{S}=\min \left\{j: \sum_{i=1}^{j} w_{i} x_{i} \geq k\right\}
$$

Then we can establish that the mixed distribution can be solved following a property established by Dantzig (1957), for the case of a continuous knapsack problem which can be stated as follows:

The optimal solution $\bar{x}$ of continuous knapsack problem is

$\bar{x}_{j}=1$ for $\mathrm{j}=1, \ldots, \mathrm{s}-1$

$\bar{x}_{j}=0$ for $\mathrm{j}=\mathrm{s}+1, \ldots, \mathrm{n}$

$\bar{x}_{s}=\frac{\bar{c}}{w_{s}} \quad$ where $\quad \bar{c}=c-\sum_{j}^{s-1} w_{j}$ (where $\mathrm{c}$ in this case represents the capacity of the knapsack )

Going by Dantzig(1957), it is observed that the problem is a $0-1$ knapsack problem. Since we are considering on a bounded problem, we take our upper bound to be $d$, thus our optimal solution is

$$
\begin{aligned}
& \bar{x}_{i}=d_{i} \text { for } \mathrm{i}=1, \ldots, \mathrm{s}-1 \\
& \bar{x}_{i}=0 \text { for } \mathrm{i}=\mathrm{s}+1, \ldots, \mathrm{n} \\
& \bar{x}_{s}=\frac{\bar{c}}{w_{s}} \quad \text { where } \bar{c}=k-\sum_{j}^{s-1} w_{j}
\end{aligned}
$$

$\operatorname{Min} \mathrm{f}=$

$\sum_{i=1}^{n} C_{i} x_{i}+g \int_{Q}^{U} P(k)\left(k-\sum_{i=1}^{n} w_{i} x_{i}\right) d k+h \int_{L}^{Q} P(k)\left(\sum_{i=1}^{n} w_{i} x_{i}-k\right) d k$

$$
=
$$

$\left.\left.\sum_{i=1}^{n} C X_{i}+g \int_{Q}^{U} k(p) d k-g \int_{Q}^{U} P(k) \sum_{i=1}^{n} w_{i} X_{i}\right) d k+h \int_{L}^{Q} P(k) \sum_{i=1}^{n} w_{i} X_{i}\right) d k-h \int_{L}^{Q} k(f k) d k$

S.t $\quad \sum_{i=1}^{n} w_{i} x_{i} \geq Q$

$$
\sum_{i=1}^{n} w_{i} x_{i}<Q+\Delta Q
$$

$0 \leq x_{i} \leq d_{i} ; \quad x_{i} \geq 0$

$\operatorname{Min} \mathrm{f}=$

$\sum_{i=1}^{n} C_{i} x_{i}+g \int_{Q}^{U} P(k)\left(k-\sum_{i=1}^{n} w_{i} x_{i}\right) d k+h \int_{L}^{Q} P(k)\left(\sum_{i=1}^{n} w_{i} x_{i}-k\right) d k$

$=$

$\left.\left.\sum_{i=1}^{n} G X_{i}+g \int_{Q}^{U} k(f) d k-g \int_{Q}^{U} P(k) \sum_{i=1}^{n} \psi_{i} x_{i}\right) d k+h \int_{L}^{Q} P(k) \sum_{i=1}^{n} w_{i} x_{i}\right) d k-h \int_{L}^{Q} k(f) d k$

(Here, we make $\mathrm{P}(\mathrm{k})=\mathrm{P}(\mathrm{x})$, such that

$\left.\int P(k) d k=\int P(x) d x\right)$

$\left.\left.=\sum_{i=1}^{n} G x_{i}+g \int_{Q}^{U} x \not(x) d x-g \int_{Q}^{U} P(x) \sum_{i=1}^{n} W_{i} x_{i}\right) d x+h \int_{L}^{Q} P(x) \sum_{i=1}^{n} W_{i} x_{i}\right) d x-h \int_{L}^{Q} x(x) d x$

$=\sum_{i=1}^{n} C x_{i}+g \int_{Q}^{U}\left(\frac{\Gamma(x+2)}{x(x+1)^{x}(x+1)^{2}}\right) d x-g \int_{Q}^{U}\left(\frac{\Gamma(x+2)}{x(x+1)^{x}(x+1)^{2}}\right)\left(\sum_{i=1}^{n} w_{i} x_{i}\right) d \lambda$

$+h \int_{L}^{Q}\left(\frac{\Gamma(x+2)}{x !(x+1)^{x}(x+1)^{2}}\right)\left(\sum_{i=1}^{n} w_{i} x_{i}\right) d x-h \int_{L}^{Q} x\left(\frac{\Gamma(x+2)}{x !(x+1)^{x}(x+2)^{2}}\right) d x$

S.t $\sum_{i=1}^{n} w_{i} x_{i} \geq Q$

$$
\sum_{i=1}^{n} w_{i} x_{i}<Q+\Delta Q
$$

$0 \leq x_{i} \leq d_{i} ; \quad x_{i} \geq 0$

\section{CONCLUSION:}

This paper has considered a stochastic knapsack 
problem with the capacity following a mixture of of Poisson and Exponential distribution. The graph of the distribution is an indication that it is a decreasing function, since the graph decreases as the corresponding values of ' $x$ ' increases. The algebraic solution to the problem is provided.

\section{REFERENCES}

BALAS, E. AND ZEMEL, E. (1980). An algorithm for large zero-one knapsack problems. Operations Research 28: 1130-1154

BEAN, J. C., NOON, C. E., SALTON G. J. (1987). Asset divestiture at Homart Development Company. Interfaces, 17(1):48-64

BELLMAN,R.(1957)Dynamic Programming, Princeton, N.J: Princeton University Press

BENLI, O.S \& BILICI,H. (2006) Capital Budgeting with risk adjustment for large scale interdependent projects. Review of social, Economic \& Business studies, Vol. 7/8, 71-82

BERNHARD, R.H. (1969) Mathematical Programming Models for Capital Budgeting- a survey, generalization, and critique. Journal of Financial and Quantitative Analysis 4 (2): 111-158

CABOT, A. V.(1970). An Enumeration Algorithm for knapsack problems. Operations Research 18; 306-311

CARRAWAY, R. L., SCHMIDT, R. L. AND WEATHERFORD, L. R. (1993). An Algorithm for Maximizing Target Achievement in the Stochastic Knapsack Problem with Normal Returns. Naval Research Logistics Quarterly, 40, 161-173

DANTZIG, G.B (1957). Discrete variable extremum problems. Operations Research 5:266-277

FAYARD, D. AND PLATEAU, G.(1975). Resolution of the 0-1 knapsack problem: comparison of methods. Mathematical Programming, 8:272-307

FELLER, WILLIAM (1970) An Introduction to Probability theory and its Application, Vol. $13^{\text {rd }}$ Edition John Wiley and Sons

FREELAND, J.R AND RONSENBLATT, M.J (1978) An Analysis of Linear Programming formulations for the Capital Rationing problem. The Engineering Economist 23: 49-61.

GREENE, W.H (2003) Econometric Analysis $5^{\text {th }}$ Edition

HALL, N .G., HERSHEY, J.C., KESSLER, L.G., SHOTTS, R.C. (1992). A model for making project funding decision at The National Cancer Institute. Operations Research, 40(6): 1040-1052
HENIG, M.I,(1990) "Risk Criteria in the Stochastic Knapsack Problem", Operations Research, 38, 820825

HOROWITZ, E. AND SAHNI, S. (1974). Computing Partitions with application to the knapsack problem. Journal of ACM.21:277-292

KELLERER, H., PFERSCHY, U. \& PISINGER, D. (2004)Knapsack problems. Berlin springer.

KLEYWEGT, A. J. \& PAPASTAVROU J.D.(1998). The dynamic and stochastic knapsack problem. Operations Research 46, 17-35

KOLESAR, P.J (1967).A branch and bound algorithm for the knapsack problem. Management Science, 13:723735

KRESS M., PENN M., AND POLUKAROV M. (2007). The Minmax Multidimensional Knapsack Problem with Application to a Chance-Constrained Problem. Wiley Periodicals, Inc. Naval Research Logistics 54, 656-666

LORIE J.H., AND SAVAGE C.J., (1955). Three problems in Rationing Capital. Journal of Business 28(4) 229-239

MARTELLO,S. AND TOTH, P. (1977). An upper bound for the 0-1 knapsack problem and a branch and bound algorithm, European Journal of operational Research. 1:169-175

MARTELLO,S. AND TOTH, P.(1988). A new algorithm for the 0-1 knapsack problem, Management Science 34: 633-644

MARTELLO,S. AND TOTH, P.(1990). Knapsack Problems, Algorithms and Computer Implementations. John Wiley \& sons New York

MATHUR, K.(1980). An enumerative algorithm for the general multiple-choice knapsack problem. Ph.D Thesis, Case Western Reserve University.

MEYER, P.L. (1965) Introduction Probability and Statistical Application $2^{\text {nd }}$ Edition.

MOOD, A., GRAYBILL, F. AND BOES, D.(2006) Introduction to the Theory of Statistics. $3^{\text {rd }}$ Edition,McGraw-Hill.

MORITA, H., ISHII, H. AND NISHIDA, T., (1989) “ Stochastic Linear Knapsack Programming Problem and its application to a Portfolio Selection Problem," European Journal of Operations Research,40 329-336

MUNAPO, E. (2007). The Variable Sequencing Approach: A solution to the capital Budget Allocation Model. Advances in Applied Mathematical Analysis 2, 15-30

MUNAPO, E.(2007). The variable sequencing approach: A solution to the capital budget allocation model. Advances in Applied mathematical analysis.

MURTY, K. G (1995). Operations Research: Deterministic optimization models. Prentice Hall, N. J. 
MYERS, S.C. (1972) A note on Linear Programming and Capital Budgeting. The Journal of Finance, 27: 89-92.

NAUSS, R.M (1976).An efficient algorithm for the 0-1 knapsack problem. Management Science. 23:27-31

NERC (1975). Natural Environment Research Council. Flood studies Report 1, Hydrologic Studies. Whitefriars Press Ltd., London, United Kingdom.

PAPASTAVROU, J. D., RAJAGOPALAN, S., KLEYWEGT, A.J., (1996) "Discrete Dynamic Programming and Capital Allocation,” Management Science, 42, 17061718

PARK, C.S. AND SHARP-BETTE G.P. (1990) Advanced Engineering Economics. John Wiley and sons, N.Y

RAM, B. AND SARIN, S. (1988). An algorithm for the 0-1 equality knapsack problem. The Journal of Operations Research Society, 39:1045-1049

RAYNAL J. AND GUEVARA J.(1997) Maximum Likelihood Estimators for the two Populations Gumbel distribution. Hydrological Science and Technology Journal, 13(14) $47-56$

ROSS, K. W. AND TSANG, D. H. K., (1989) "The Stochastic Knapsack Problem," IEEE Transactions on Communications,37, 740-747

SHAPIRO, J.F. (1968). shortest Route methods for finite state space Deterministic Dynamic Programming Problems. SIAM Journal on Applied Mathematics. 16: $1232-1250$

SHARMA, J. K (2007) Operations Research, Theory and Applications $3^{\text {rd }}$ Edition Macmillan India Press.

SHELDON M.ROSS(1989) Introduction to Probability models $4^{\text {th }}$ Edition, Academic Press New York

STEINBERG, E. and PARKS, M.S (1979). A preference order Dynamic Program for a Knapsack Problem with
Stochastic Reward. Journal of operational Research Society,30, 141-147

STIRZAKER, DAVID (1994) Elementary Probability, Cambridge University Press, Cambridge.

VASQUEZ, M \& HAO, J. K (2001a) A logic constrained knapsack formulation and a tabu algorithm for the daily photograph scheduling of an earth observation satellite. Comp. Optim. Appl., 20, 137-157.

VOLGENANT, A \& MARSMAN, S. (1998). A core approach to the $0-1$ equality knapsack problem. Joun. Of oper. Res. Soc., 49, 86-92.

WEINGARTNER, H.M (1963) Mathematical Programming And the analysis of Capital Budgeting Problems. Prentice Hall, N.J.

WEINGARTNER, H.M (1966) Capital Budgeting of Interrelated Projects: Survey and synthesis. Management Science 12: 485-516

WILBAUT, C., SAID HANAFI \& SAD SALHI (2008):A survey of effective heuristics and their their application to a variety of knapsack problems. IMA journal of management Mathematics 19, 227-244

WITCHAKUL S. AND AYUDTHAYA P.S. (2005) Stochastic knapsack problem with continuous random capacity. Journal of Research in Engineering and Technology Vol 2(4)

WITCHAKUL S. AND AYUDTHAYA P.S. (2007) A heuristic for solving a stochastic knapsack problem with discrete random capacity. Thammasat Int. J. Sc. Tech., Vol. 12, No.1

WITCHAKUL S. AND AYUDTHAYA P.S. AND CHARNSETHIKUL, P.(2008) A stochastic knapsack problem with continuous random capacity. Journal of Mathematics and Statistics 4 (4): 269-276 